\title{
Die Wirkung von Ohrakupunktur auf verschiedene Schielformen und neuroophthalmologische Erkrankungen
}

\author{
Fritz Bieri \\ Jona, Schweiz
}

\section{Schlüsselwörter}

Schielen · Strabismus · Doppelbilder · Neuroophthalmologische

Erkrankungen · Ohrakupunktur

\section{Zusammenfassung}

Normalerweise führt die Fusion der Seheindrücke beider Augen zu einem Bild. Wenn nun im Falle einer Störung der Zusammenarbeit der äusseren Augenmuskeln der Gleichlauf beider Augen gestört ist, kommt es zu Doppelbildern oder zu einer sensorischen Anpassung, einer Amblyopie. Mit Akupunktur bezeichnet man die Stimulation peripherer Körperpunkte durch Akupunktur- oder Lasernadeln. In dieser Arbeit wurde mit Ohrakupunktur und Laserakupunktur gearbeitet. Zur Behandlung von Schielproblemen mit Akupunktur: In der Traditionellen Chinesischen Medizin (TCM) soll mit verschiedenen therapeutischen Massnahmen wie Akupunktur das Ungleichgewicht zwischen yin und yang, das der Krankheit zugrunde liegt, wieder in ein Gleichgewicht zurückgeführt werden, und die Säfte sollen wieder ungehindert zirkulieren können. Lähmungsschielen wird in der TCM als «vom Wind hervorgerufenes Schielen» bezeichnet. Der Wind wird durch verschiedene Ursachen hervorgerufen: Der Funktionskreis Leber, Milz oder Niere kann Ausgangspunkt der Störung sein, die sich schlussendlich als Wind in der oberen Körperhälfte äussert. Der Wind ist Ausdruck eines Ungleichgewichts im Patienten und kann gemäss chinesischer Lehre die Leitbahnen im Bereich des Auges und damit die Bewegung der Augen beeinträchtigen. Neben der klassischen Akupunktur gibt es eine moderne Entwicklung. Hierbei geht es auch um die Objektivierung und Erklärung der Wirkung von Akupunktur. Die hier vorgestellte Methode wurde noch nicht beschrieben. Die konservative Schielbehandlung mit Brillen und Abdecken eines Auges wirkt im günstigen Fall nach Wochen bis Monaten. Die schnelle Wirkung von Akupunktur auf Schielen, wie in dieser Arbeit anhand von 22 Fallbeispielen gezeigt, ermöglicht die Objektivierung der Wirkung von Akupunktur durch einfache Beobachtung.

\section{Keywords \\ Squint - Strabism · Double vision - Neuroophthalmologic disorders . Ear acupuncture}

\section{Summary}

The Effect of Ear Acupuncture in Squint and Neuroophthalmologic Disorders

Normally, double vision can be turned to single vison by fusion of the perception of both eyes. When the action of the external muscles of the eye, and therefore the concomitant movement of both eyes, is disturbed, the fusion is also disturbed and double vision or sensory adaptation (amblyopia) occurs. Acupuncture represents the stimulation of peripheral points of the body by acupuncture needles or laser needles. This paper shows the effect of ear acupuncture and laser acupuncture. Regarding the treatment of squint with acupuncture: The treatment with traditional Chinese medicine (TCM) restores the balance between yin and yang, and the fluids can circulate again. In TCM, paralytic squint is described as «squint caused by wind». Wind is the consequence of several underlying causes. The orbs liver, spleen, and kidney can be the source of the disturbance, leading to wind in the upper body. Wind is caused by imbalance, and it can block the circulation of the fluids in the region of the eyes; consequently, the movement of the eyes is disturbed. Nowadays, in addition to classic acupuncture, modern ways of acupuncture have been introduced. These also focus on explaining the effectiveness of acupuncture. The method presented here has not been described yet. The therapy of squint with glasses or occlusion ideally has an effect after weeks or even months. The fast effect of acupuncture, as shown by 22 case reports in this paper, allows to objectify the effect of acupuncture by simple observation.

\section{KARGER \\ Fax +497614520714 Information@Karger.d} www.karger.com
Dr. med. Fritz Bieri

Johannisbergstrasse 69, 8645 Jona, Schweiz

Tel. +41552123654

Fritz.Bieri@rappijona.ch 


\section{Mots-clés}

Loucher - Strabisme - Diplopie - Maladies neuro-ophtalmologiques . Acupuncture auriculaire

\section{Résumé}

Effet de l'acupuncture auriculaire sur différentes formes de strabisme et maladies neuro-ophtalmologiques

La fusion des images fournies par les deux yeux donne normalement naissance à une image unique. Dans le cas d'un trouble de la coordination des muscles oculaires externes, la perturbation du synchronisme des deux yeux entraîne une diplopie ou une adaptation sensorielle, appelée amblyopie. L'acupuncture désigne la stimulation de points corporels périphériques par des aiguilles d'acupuncture ou par le laser. Dans ce travail, l'acupuncture auriculaire et l'acupuncture au laser ont été appliquées. Au sujet du traitement de problèmes de strabisme par acupuncture: en médecine traditionnelle chinoise (MTC), différentes mesures thérapeutiques telles que l'acupuncture doivent corriger le déséquilibre entre le yin et le yang à l'origine de la maladie et rétablir un équilibre; les fluides doivent à nouveau pouvoir circuler librement. En MTC, le strabisme paralytique est qualifié de «strabisme provoqué par le vent». II existe différentes raisons à ce vent: le circuit fonctionnel du foie, de la rate ou des reins peut constituer le point de départ du trouble qui se traduit finalement dans la moitié supérieure du corps par du vent. Le vent reflète un déséquilibre chez le patient et peut, selon la théorie chinoise, affecter les voies de circulation dans la région de l'œil et ainsi le mouvement des yeux. Outre l'acupuncture classique, il existe un développement moderne de l'acupuncture. II s'agit alors aussi d'objectiver et d'expliquer l'effet de l'acupuncture. La méthode présentée ici n'a pas encore été décrite. Le traitement classique du strabisme à l'aide de lunettes et du recouvrement d'un œil est efficace après des semaines voire des mois, dans le meilleur des cas. Comme le montre ce travail en s'appuyant sur 22 exemples de cas, l'effet rapide de l'acupuncture sur le strabisme permet d'objectiver l'effet de l'acupuncture par simple observation.

\section{Einleitung}

Die Beschwerden bei Schielen (Doppelbilder, Asthenopie) kann man mit der konventionellen westlichen Methode im Wesentlichen operativ oder mit Prismen in der Brille behandeln. Oft führt die Behandlung nicht zur Beschwerdefreiheit. Bei Paresen kann man versuchen, die Doppelbilder mit einer Prismenbrille zu bessern. Durch die Okklusion des guten Auges kann versucht werden, am amblyopen Auge eine zentrale Fixation und damit eine bessere Seh-

Ohrakupunktur bei Schielfällen schärfe zu erzielen. Nach operativer Therapie beobachtet man manchmal Rezidive. Da keine Nebenwirkung befürchtet wurde, erwog man bei einigen Patienten die Therapie mit Akupunktur. Die Methode ist einfach. Die vorgestellte Akupunktur stellt eine moderne Variante der Ohrakupunktur dar und wurde im Zusammenhang mit der Behandlung von Schielproblemen noch nie vorgestellt. Bei der Anwendung von Akupunktur sind wenige Nebenwirkungen zu erwarten; dennoch muss man diese kennen. Zudem liegen neuere Arbeiten über die Behandlung von Schielproblemen mithilfe der Akupunktur vor [1-12].

Mit bildgebenden Verfahren konnte gezeigt werden, dass eine periphere Reizung, wie durch Akupunktur verursacht, innerhalb von Minuten eine Änderung der Durchblutung im Gehirn auslöst [13-43]. Damit konnte die Wirkung von Akupunktur auf das zentralnervöse System objektiviert werden. Diese Befunde ermöglichten aber nicht, die Wirkungsweise der Akupunktur zu erklären [44-46].

Der schnelle Eintritt der Wirkung auf das Schielen unter Akupunktur ermöglichte hingegen, ähnlich wie bei der Beobachtung der Durchblutungsverhältnisse unter Akupunktur, die Objektivierung der Wirkung von Akupunktur.

\section{Patienten und Methode}

Eine konsekutive, nicht randomisierte Serie von Patienten mit unterschied lichen Schielformen und neuroophthalmologischen Erkrankungen wurde mit Ohrakupunktur oder mit Laserakupunktur behandelt. Bei einer Serie von 22 konsekutiven Patienten mit unterschiedlichen Schielsyndromen und neuroophthalmologischen Erkrankungen wurde eine Akupunktur oder eine Laserakupunktur am Ohr gegeben (Tab. 1). Drei Punkte wurden gegeben: ventral kranial am Ohrläppchen, am Antitragus, $5 \mathrm{~mm}$ kaudal sowie $2 \mathrm{~mm}$ dorsal des Antitragus. Es wurde meist nur eine Nadel gegeben Der Schielwinkel, der Nystagmus und die Fixation wurden vor, während und nach der Akupunktur beobachtet. Ebenfalls wurden auch die subjektiven Angaben, wie zum Beispiel von Doppelbildern, beobachtet. Wenn ein Nystagmus bestand, wurde dieser bei verschiedenen Blickrichtungen beobachtet. 14 Patienten wurden daraufhin mit Ohrakupunktur anhand einer Nadel von 0,2 mm Dicke behandelt; diese wurde an eine Stelle am Ohrläppchen eingestochen (Abb. 1). Die Akupunktur erfolgte bei den meisten Sitzungen einseitig. Die Einwirkung dauerte etwa 20-30 min 8 Patienten wurden mit Laserakupunktur behandelt. Bei der Laseraku punktur wurde mit einem Glasfaserkabel ein Laserlicht auf eine Stelle am Ohr übertragen. Mit dem Laserlicht $\left(585 \mathrm{~nm}, 5-10 \mathrm{~W} / \mathrm{cm}^{2}\right)$ wurde eine Frequenz übertragen. Das Tastverhältnis des Signals betrug 50\%.

Die Beobachtung des Patienten erfolgte unmittelbar nach Beginn der Behandlung und dann im Abstand von 5 min. Die Behandlung wurde nach wenigen Tagen wiederholt. Während der Behandlung lag der Patient. Wenn möglich, wurde der Patient nach Monaten oder Jahren über die Wirkung der Behandlung erneut befragt

Diagnosen: 14 Patienten wurden aufgrund von konkomitierendem Schielen (4-12, 14-16, 20, 21), 12 wegen Auswärtsschielen (4-9, 11, 12, 14 $15,17,20)$ und 9 wegen Amblyopie $(6,7,9-12,14-16)$ behandelt (Mehrfachdiagnosen möglich). 4 Patienten hatten eine neuroophthalmologische Erkrankung $(1-3,13)$; davon hatten 3 Patienten eine multiple Sklerose (MS) (1-3), und eine Patientin hatte ein Aneurysma (13). Eine Patientin hatte eine beidseitige Amblyopie (10) und eine ein Schädeltrauma (17); dreimal lag ein Nystagmus vor (bei 2 Patienten mit MS und bei der Patientin mit einem Aneurysma (2, 3, 13). 

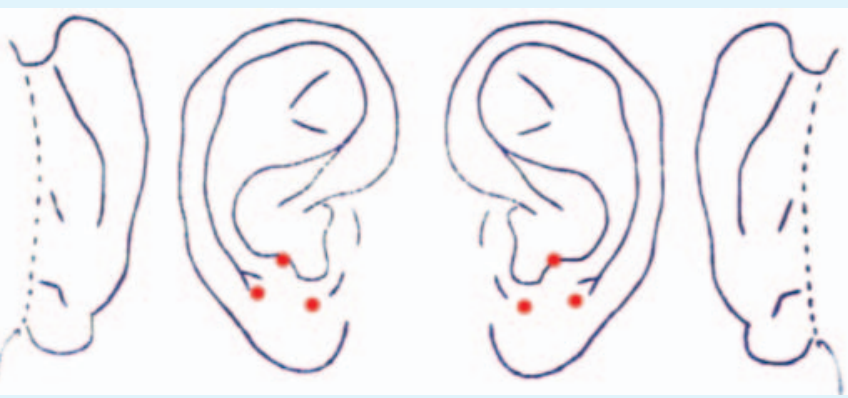

Fallberichte

Fall 1

31-jähriger Patient mit Trochlearisparese rechts. Der Patient hatte seit 10 Tagen Doppelbilder. Diagnose: Trochlearisparese rechts, paralytisches Schielen mit vertikalen Doppelbildern. In der Folge wurde die Diagnose einer MS gestellt. Befunde vor der Therapie: Der bestkorrigierte Visus war beidseits voll. Die Doppelbilder traten bei Blick geradeaus und nach unten auf. Therapie: Ins rechte Ohr wurde eine Akupunkturnadel gegeben. Ergebnis: Während der Akupunktur gab der Patient an, die Doppelbilder hätten abgenommen. Zweimalige Ohrakupunktur führte zu einer deutlichen Besserung der Doppelbilder. Bei Blickrichtung geradeaus sah der

Abb. 1. Ohrakupunkturpunkte am Ohrläppchen.

Tab. 1. Wirkung der Akupunktur oder Laserakupunktur am Ohr bei 22 Patienten mit unterschiedlichen Schielsyndromen und neuroophthalmologischen Erkrankungen

\begin{tabular}{llll}
\hline Fall & Diagnose & Therapie & Ergebnis \\
\hline 1 & Trochlearisparese rechts, MS & Ohrakupunktur & Besserung \\
2 & Internukleäre Ophthalmoplegie rechts, MS & Ohrakupunktur & Besserung \\
3 & Internukleäre Ophthalmoplegie beidseits, MS & Ohrakupunktur & Besserung \\
4 & Dekompensierende Exophorie & Laserakupunktur rechtes Ohr 20 Hz & Besserung \\
5 & Dekompensierende Exophorie, Linksfixation & Laserakupunktur rechtes Ohr 20 Hz & Besserung
\end{tabular}

6 Strabismus divergens monolateralis links, Amblyopie links

7 Strabismus divergens monolateralis links, Amblyopie links

8 Strabismus divergens monolateralis rechts, Status nach Schieloperation, Höhenschielen

9 Strabismus divergens monolateralis rechts, Anisomyopie und Amblyopie rechts

10 Nanophthalmus beidseits (hochgradige Dertsichtigkeit), Amblyopie beidseits, Strabismus divergens monolateralis rechts

10 Beidseitige Amblyopie bei Nanophthalmus und Astigmatismus, Strabismus divergens monolateralis rechts

11 Strabismus intermittens divergens rechts, Amblyopie Laserakupunktur am rechten $\mathrm{Ohr} 0,20 \mathrm{~Hz}$ rechts, Status nach vier Schieloperationen

12 Anisometropie, Strabismus divergens monolateralis links, Status nach Schieloperation, Amblyopie links

13 Status nach Embolisation eines Aneurysmas der Arteria vertebralis, vertikale Doppelbilder, vertikaler blickrichtungsabhängiger Nystagmus

14 Strabismus divergens monolateralis rechts, Amblyopie rechts

Laserakupunktur rechtes $\mathrm{Ohr} 7,8 \mathrm{~Hz}, 10 \mathrm{~Hz}$

Laserakupunktur Punkt B2 (Nasenwurzel)

Ohrakupunktur rechts

Laserakupunktur rechtes $\mathrm{Ohr}$

Laserakupunktur Punkt B2 mit $20 \mathrm{~Hz}$

am rechten Auge nasal $10 \mathrm{~Hz}$,

lateral $299,75 \mathrm{~Hz}$

Ohrakupunktur am Antitragus beidseits

Ohrakupunktur rechts insgesamt fünfmal

Laserakupunktur am rechten Ohr mit $20 \mathrm{~Hz}$

Laserakupunktur am rechten Ohr mit $20 \mathrm{~Hz}$

Amblyopie links, hochgradiger Astigmat
Strabismus divergens monolateralis links

16 Amblyopie links, Astigmatismus links, exzentrische Fixation links

17 Commotio und contusio cerebri frontotemporal vor 4 Jahren, Doppelbilder seit 6 Monaten, Dekompensierende Exophorie

18 Abduzensparese rechts seit 2 Tagen

19 Abduzensparese rechts seit 2 Tagen, insulinpflichtiger Diabetes mellitus

20 Dekompensierende Exophorie, Asthenopie

21 Trochlearisparese rechts

22 Abduzensparese rechts seit 3 Tagen

MS = Multiple Sklerose.
Ohrakupunktur rechts

Ohrakupunktur rechts

Ohrakupunktur rechts

Ohrakupunktur rechts

Ohrakupunktur rechts

Ohrakupunktur rechts

Ohrakupunktur rechts
Besserung mit $10 \mathrm{~Hz}$, nicht mit 7,8 Hz

Besserung mit $5672 \mathrm{~Hz}$

Besserung unter der Nadel

Besserung unter $9592 \mathrm{~Hz}$

Besserung

Besserung

Besserung mit 559,5 Hz und $9592 \mathrm{~Hz}$ an gleicher Stelle; keine Besserung Besserung während der Akupunktur

anhaltende Besserung während etwa einer Woche

Besserung; die Fixation war während der Belichtung zentral; mit $10 \mathrm{~Hz}$ und $40 \mathrm{~Hz}$ an gleicher Stelle trat keine Besserung auf

Besserung; die Fixation war während der Belichtung zentral

Besserung; die Fixation war während der Belichtung zentral

Besserung während 2-3 Wochen

keine Besserung

keine Besserung

Besserung

Besserung

keine Besserung 
war schon unter der Ohrakupunktur grösser. Die subjektive Verbesserung der Doppelbilder dauerte nach der Behandlung etwa 5 Tage an.

\section{Fall 2}

Der 28-jährige Patient bemerkte Doppelbilder besonders bei Blick nach rechts. Eine MS war bekannt. Befunde: Fernvisus: rechts $-2,0$, kombiniert $-1,25$, Achse $25^{\circ}=0,8$; links mit $-3,0=1,0$ partim. Folge bewegungen wurden ohne Einschränkung ausgeführt. Im Kopfneigetest zeigte sich eine Höherstellung links. Binokularsehen war im Schober-Test nachweisbar. Das Gesichtsfeld war beidseits voll (Octopus-G2-Programm) Isokorie, keine afferente Pupillenstörung. Druck beidseits $17 \mathrm{~mm} \mathrm{Hg}$ (Normbereich). Die Doppelbilder bildeten sich spontan zurück.

Der Patient meldete sich 5 Jahre später wieder. Er hatte seit 3 Jahren gelegentlich Doppelbilder. Befund: Korrigierter Fernvisus: rechts 0,6; links 1,0 partim. Nahvisus: rechts 0,3 ; links 0,6 . Druck beidseits $20 \mathrm{~mm} \mathrm{Hg}$ Sehschulstatus: Keine Einstellbewegung für Ferne und Nähe im Abdecktest. Leichter Endstellungsnystagmus vor allem bei Blick nach links. Konver genz positiv. Bagolini: Für die Nähe und Ferne positiv. Maddox-Rotglas: rechts 1 Prismendioptrie (PD) eso; links 1 PD eso. Stereotest nach Lang: positiv. Titmus-Stereotest: Ringe 1-9 positiv, Tiere A, B und C positiv. Im Hess-Test sah man keine sichere Abweichung. Das Gesichtsfeld war beid seits normal. Bei der Prüfung der Sakkaden hatte man den Eindruck, das rechte Auge bewege sich verlangsamt. Therapie: Eine erste Ohrakupunktur hatte subjektiv nach 3 Tagen wenig Auswirkung. Allerdings war kein Endstellungsnystagmus mehr festzustellen (den Endstellungsnystagmus sieht man bei Blick nach lateral). Nach der zweiten Ohrakupunktur war das Sehen subjektiv deutlich besser. Nach der dritten Ohrakupunktur gab der Patient an, die Doppelbilder hätten sich gebessert.

Bericht der neurologischen Poliklinik: Encephalomyelitis disseminata mit schubförmigem und oligosymptomatischem Verlauf seit 18 Jahren. Durchschnittliche Schubrate seit 6 Jahren einmal pro Jahr. Aktueller Expanded Disability Status Score (EDSS) 3,00. Interferon beta-1b von 1998 bis März 2000, dann Umstellung auf Glatirameracetat. Nach der vierten Ohrakupunktur gab der Patient eine deutliche Besserung der Doppelbilder an.

Neuroophthalmologische Diagnose der Augenklinik: Status nach internukleärer Ophthalmoplegie rechts, wobei die Adduktionssakkaden am rechten Auge stark verlangsamt waren. Gleichzeitig auch ein dissoziierter Nystagmus am linken Auge gegen lateral bei Blickwendung nach links. Die Ishihara-Tafeln zeigten eine deutliche Deutanstörung an; man nahm eine angeborene Farbsinnstörung beidseits an. Die Stereopsis war normal. Kein Uhthoffsches Symptom bei körperlicher Betätigung. Visuell evozierte Potenziale: Am linken Auge waren die Latenzzeiten unauffällig Am rechten Auge waren die Amplituden gesamthaft etwas niedriger im Sinne einer leichten Axonreduktion; zudem waren die Latenzzeiten mässig verspätet; für die 40 Winkelminuten-Schachbrettmuster betrug die Latenzzeit 106-107 ms; Norm: um 90 ms. Ähnliche Verspätungen bestanden auch mit den kleineren Schachbrettmustern.

4 Monate später kam der Patient wieder, weil er manchmal Doppelbilder hatte. Er gab an, dass sich die Doppelbilder jeweils nach 1-2 Wochen nach der Ohrakupunktur wieder eingestellt hätten. Therapie: Erneut wurde eine Ohrakupunktur vorgenommen. 6 Wochen später gab er an, die Doppelbilder seien wesentlich besser. Er verspüre auch nicht mehr das Zittern der Augen. Subjektiv sah er mit dem rechten Auge schlechter. Endstellungsnystagmus beidseits. Therapie: Ohrakupunktur.

5 Wochen später hatten sich die Doppelbilder wieder eingestellt. Therapie: Ohrakupunktur besserte die Doppelbilder noch während der Behandlung. 5 Wochen später nahm er wieder Doppelbilder bei Blick nach links wahr. Therapie: Ohrakupunktur. 6 Wochen später gab er wiederum an, die Doppelbilder seien unter Akupunktur deutlich besser gewesen. Befund: Endstellungsnystagmus nach lateral beidseits. Therapie: Ohr akupunktur. Befund nach 13 Tagen: Kein Nystagmus feststellbar, in allen Blickrichtungen. Das Gesichtsfeld war beidseits voll (Octopus G2). Der Patient erhielt eine Medikation mit Glatirameracetat. 7 Wochen später hat te er wiederum Doppelbilder. In der Zwischenzeit wurde eine systemische Therapie mit Steroiden durchgeführt; die Sehstörung wurde damit nicht besser. Befunde: Der Fernvisus war mit eigener Brille rechts 0,7 knapp und links 1,0 knapp. Im Kopfneigetest nach rechts zeigte der Patient einen Höherstand des linken Auges, bei Neigung nach links einen Höherstand des rechten Auges.
6 Wochen später hatte man wegen einem Schub erneut eine Behandlung mit systemischen Steroiden durchgeführt. Doppelbilder waren noch vor handen. Befund: Höherstellung links beim Kopfneigetest mit Neigung nach links. Therapie: Ohrakupunktur.

2 Wochen später hatte der Patient noch Doppelbilder. Befund: Bei Kopfneigung nach links bestand eine Höherstellung links über rechts. Therapie: Ohrakupunktur. Noch während der Ohrakupunktur wurden die Doppelbilder deutlich besser und der Nystagmus bei Blick nach links verschwand

Drei Wochen später hatte der Patient wieder Doppelbilder. Der Patient beobachtete eine Sensibilitätsstörung am linken Bein. Befunde: Gerad stand im Schober-Test. Therapie: Ohrakupunktur. Nach 7 Wochen hatte der Patient wieder Doppelbilder im Kopfneigetest. Befund: Höherstand links über rechts. Therapie: Ohrakupunktur.

Einen Monat später waren die Doppelbilder besser. Der Patient hatte Glatirameracetat eingenommen. Befund: Der Kopfneigetest fiel weniger deutlich aus. Therapie: Ohrakupunktur.

8 Wochen später waren die Doppelbilder nicht sehr störend. Befund: Im Kopfneigetest bestand noch ein Höherstand links. 2 Jahre später ergab ein telefonisches Gespräch, die Akupunktur habe jeweils genützt, die Dop pelbilder hätten sich noch während der Behandlung gebessert oder seien verschwunden und der Effekt hätte jeweils etwa eine Woche angehalten Der Patient hatte aktuell keine Doppelbilder mehr.

Fazit: Bei diesem Patienten mit einer internukleären Ophthalmoplegie im Rahmen einer MS konnte die Ohrakupunktur die Doppelbilder wäh rend einiger Tage bessern. Die Besserung trat nach Angabe des Patienten schon während der Ohrakupunktur ein.

\section{Fall 3}

46-jährige Patientin, bei der eine MS bekannt war. Anamnese: 1971 wurde eine Retrobulbärneuritis festgestellt. 1978 erfolgte die Diagnose einer Optikusneuropathie beidseits. Erstkonsultation 1983. Befunde: Der Visus war beidseits voll. Nahvisus mit Nahaddition rechts 0,6 und links 0,7 . Im Sehschulstatus fiel rechts eine Einschränkung der Beweglichkeit der Augen nach temporal und nasal auf. Endstellungsnystagmus rechts.

6 Jahre später wurde sie wieder mit Doppelbildern, die seit 2 Wochen anhielten, vorstellig. Befunde: Endstellungsnystagmus beidseits. Kopfneigetest unauffällig. Im Weiss-Test sah man keine Abweichungen der Augenbewegungen (Rot-Grün-Trennung). Beidseits leichte Einschränkung der Einwärtswendung, Endstellungsnystagmus. Die Papillen waren nun beidseits etwas blass. Pupillen: Afferenzdefizit rechts als Ausdruck eines Schadens des Nervus opticus. Die Folgebewegungen waren etwas sakkadiert. 3,5 Jahre später hatte sie wieder vermehrt Doppelbilder. Diese verschwanden nach einer Woche spontan. Einen Monat später traten erneut Doppelbilder auf. Therapie: Ohrakupunktur rechts. Die Doppelbilder bildeten sich zurück, die Müdigkeit war besser geworden. Zunehmend war das Tastgefühl an den Händen gestört.

Diagnose der Augenklinik: Verdacht auf eine erhebliche Neuropathie an beiden Nervi optici mit relativ fortgeschrittener Optikusatrophie rechts. Differentialdiagnose: Durchgemachte beidseitige internukleäre Ophthal moplegie oder eine cerebelläre Läsion. Befunde: Das Tastgefühl beider Hände war gestört. Befunde: Visuelle Potenziale: Erhebliche Leitungs störung an beiden Augen (130 ms). Die Latenzzeit der visuellen evozierten Potenziale befindet sich normalerweise zwischen 95 und $120 \mathrm{~ms}$.

Zeitweilig leicht rotatorischer Spontannystagmus vor allem am rechten Auge. Die sinusoidale Blickfolge in der Horizontalen war gestört; das ab duzierende Auge sakkadierte beidseits. Die Sakkadenauslösung erfolgte vor allem am rechten Auge gegen temporal überschiessend.

Bewegungswahrnehmung: «Motion-defined letter detection» nach Regan: Schwierigkeiten mit ganz langsam bewegten Gegenständen. Dieser Befund sprach für eine Läsion der Verbindungsbahnen zu den kortikalen Zentren MT und MST, die für die Bewegungswahrnehmung verantwort lich sind.

10 Jahre später kam die Patientin erneut in die Sprechstunde. Diagnose: Doppelbilder. Diese waren oft vertikal. In der Zwischenzeit hatte sie mehrere Rezidive von Doppelbildern. Befunde: Keine Doppelbilder bei Folgebewegung. Beidseits Endstellungsnystagmus. Keine sichere Einstel lungsbewegung im Kopfneigetest. Eingeschränkte Einwärtswendung rechts. Sie schleppte das rechte Bein nach, und es bestand eine Sensibilitätsstörung der rechten Hand. Therapie: Erstmals wurde eine Ohrakupunktur rechts durchgeführt. Ergebnisse: Noch unter den Nadeln war der 
Endstellungsnystagmus besser und gröber. Die Sensibilitätsstörung der rechten Hand war unmittelbar nach dem Einstich gut. Noch während der Akupunktur war die Gefühlsstörung nun in der linken Hand wahrnehm bar. Der Endstellungsnystagmus war beidseits gröber, besser als vor der Ohrakupunktur und erschöpfbar. Zwischenanamnese: Eine Woche später berichtete die Patientin von einer guten Wirkung der Akupunktur. Das Tastgefühl in der rechten Hand stellte sich wieder ein. Die Sehstörung sei gebessert. Therapie: Erneut erfolgte eine Ohrakupunktur rechts. Ergebnis: Eine Woche später sah sie nicht mehr doppelt. Befunde: Der Endstellungsnystagmus bestand noch beidseits. Keine Einstellbewegung im Kopfneigetest. Die Sakkaden zeigten beidseits eine eingeschränkte Einwärtswendung Therapie: Ohrakupunktur.

Befunde 4 Wochen später: Zeitweise vertikale Doppelbilder. Diese waren vor Beginn der Ohrakupunktur dauernd feststellbar; jetzt nahm sie diese vertikalen Doppelbilder noch einmal pro Tag für einen kurzen Moment wahr. Mit Blinzeln konnte sie die Doppelbilder überwinden. Der Endstellungsnystagmus war beidseits vorhanden. Therapie: Ohrakupunktur rechts. Ergebnis: Noch unter der Ohrakupunktur nahm der Endstellungsnystagmus beidseits ab. Die Einwärtswendung wurde rechts sofort besser, links jedoch nicht. Objektiv waren die Doppelbilder besser.

Befunde 2 Wochen später: Doppelbilder waren nicht mehr aufgetreten. Die Einwärtswendung war beidseits noch schwach. Endstellungsnystagmus. Therapie: Erneut Ohrakupunktur rechts. Eine telefonische Erkundigung einen Monat später ergab, dass die Patientin keine Doppelbilder mehr wahrnahm.

Ergebnisse: Diagnose: Durchgemachte beidseitige internukleäre Ophtalmoplegie oder cerebelläre Läsion im Rahmen einer fortgeschrittenen MS. Rezidivierende Episoden von Doppelbildern. Opticusneuropathie. Therapie: Ohrakupunktur. Resultate: Die Doppelbilder wurden mehrmals schnell (unter der Akupunktur) und möglicherweise dauerhaft gebessert. Die Symptome sprachen unterschiedlich an: Der Endstellungsnystagmus war gut beeinflussbar, die Einwärtswendung nicht oder nur ganz kurzfristig. Auffallend war auch das Ansprechen der Sensibilitätsstörung der rechten Hand, die sich schnell gebessert hat; gleichzeitig trat aber die Sensibilitätsstörung an der linken Hand auf. Subjektiv hat sich die Gangstörung nach Akupunktur etwas gebessert.

Wie schon bei den vorher beschriebenen Fällen gab die Patientin an die Doppelbilder hätten sich bei Ohrakupunktur regelmässig schon wäh rend der Sitzung gebessert. Gestochene Ohrpunkte: Einmal wurde eine Nadel auf dem Antitragus rechts gegeben, bei den folgenden Behandlungen jeweils eine Nadel auf dem Ohrläppchen beidseits.

Fazit aller drei Fälle mit MS (1-3): Die Doppelbilder besserten sich meist schon während der Ohrakupunktur deutlich. Ein Rezidiv trat nach Tagen bis Wochen auf. Eine Überdosierung der Wirkung wurde nicht beobachtet. Die Doppelbilder und der Nystagmus sprachen gut an. Die Einwärtswendung sprach kaum an. Auffallend war bei Fall 3, dass sich die Doppelbilder wenige Sekunden nach Beginn der Akupunktur besserten. Wegen der schnell und regelmässig einsetzenden Besserung nahmen wir an, dass die Akupunktur die Besserung verursacht hat.

\section{Fall 4}

15-jähriger Patient. Diagnose: Dekompensierende Exophorie. Befunde vor der Therapie: Der Patient sah ohne Korrektur beidseits voll. Therapie: An einem Punkt am ventralen Ohrläppchen rechts wurde mit einer Laserneedle die Frequenz $20 \mathrm{~Hz}$ gegeben. Die Sitzung wurde zweimal wiederholt Dauer jeweils 8 min. Resultate: Während der ersten Behandlung konnte man eine Besserung der Einstellbewegung beim Abdecken eines Auges beobachten. Bei der Kontrolle nach einem Monat beobachtete man spontan Geradstand; eine Einstellbewegung beim Abdecktest war nicht mehr zu beobachten. Die Besserung hielt bislang 2 Jahre an.

\section{Fall 5}

18-jährige Patientin. Diagnose: Dekompensierende Exophorie, bevorzugte Linksfixation. Geringe Myopie. Befunde vor der Behandlung: Fernvisus beidseits 1,0. Exklusion rechts bis alternierend im Bagolini-Test. Synoptophor: Objektiver Winkel -15 PD. 12 PD links über rechts. Konvergenz: Nicht möglich. Therapie: Am rechten Ohrläppchen wurde ventral eine Laserneedle mit der Frequenz $20 \mathrm{~Hz}$ gegeben (Abb. 2). Ergebnis: Die Konvergenz war nach $10 \mathrm{~s}$ bis auf $12 \mathrm{~cm}$ möglich. Die Einstellbewegung war deutlich besser und schneller, besonders links. Nach Löschen der Laser-

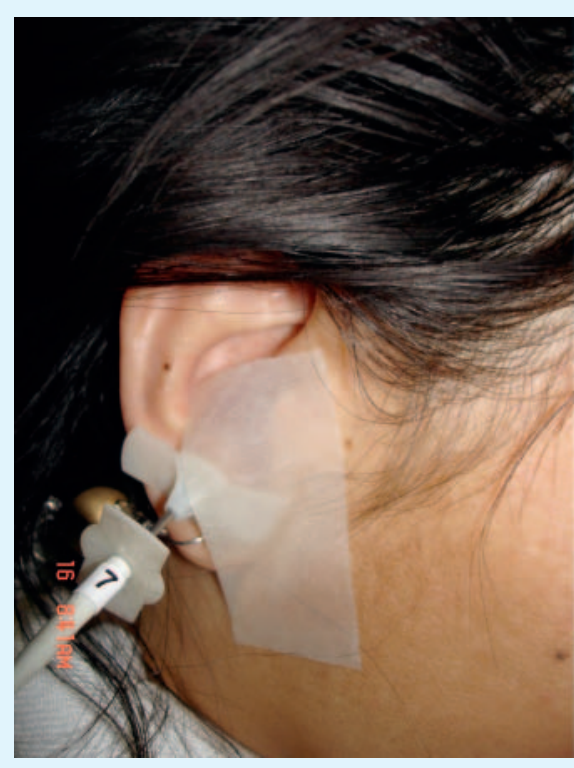

Abb. 2. Fall 5: Dekompensierende Exophorie. Am rechten Ohrläppchen wurde mit der Laserneedle eine Frequenz von $20 \mathrm{~Hz}$ gegeben

needle war die Einstellbewegung nach einigen Sekunden wieder wie vor der Belichtung mit der Laserneedle. Nachdem die Laserneedle wieder an der gleichen Stelle belichtete, war die Konvergenz wieder deutlich besser als ohne Belichtung, aber nicht mehr so gut wie bei der ersten Belichtung.

\section{Fall 6}

34-jähriger Patient. Diagnose: Strabismus divergens monolateralis links. Befunde vor der Behandlung: Refraktion: rechts $+1,0$, kombiniert $-0,5$, Achse 100; links $+2,5$, kombiniert - 0,5 , Achse 17. Fernvisus mit Korrektur rechts 1,0; links 0,4. Die Konvergenz war schwach. Verzögerte Fusions aufnahme mit einem Restwinkel von $2^{\circ}$. Therapie: Laserakupunktur am ventralen Ohrläppchen rechts. Ergebnis: Mit der Frequenz 7,8 Hz war keine Wirkung zu beobachten; mit $10 \mathrm{~Hz}$ war die Einstellbewegung deutlich kleiner. Der Schielwinkel war somit kleiner. Die Wirkung konnte noch einmal mit der gleichen Laserakupunktur beobachtet werden: Die Einstellbewegung und die Konvergenz waren unter der Belichtung deutlich besser.

Fall 7

26-jähriger Patient. Diagnose: Leichte Schielamblyopie links. Strabismus divergens monolateralis links. Befunde vor der Therapie: Fernvisus rechts 1,0; links 1,0 partim. Nahvisus rechts 0,8; links 0,6. Im Abdecktest fern stellte das rechte Auge gut zu Binokularsehen ein, das linke zögernd. The rapie: Im Bereich des Nasenrückens wurde eine Laserakupunktur mit der Frequenz von $5672 \mathrm{~Hz}$ gegeben. Ergebnisse: Die Einstellbewegung beim Abdecktest wurde deutlich besser mit einer Laserakupunktur am Nasen rücken (Punkt Blase 2) mit der Frequenz $5672 \mathrm{~Hz}$. Dies wurde einmal wiederholt, mit dem gleichen Ergebnis. Mit einer Nadel im Bereich des lateralen Lidwinkels beidseits konnte keine Wirkung auf das Schielen beobachtet werden (Punkt 1 des Gallenblasenmeridians).

\section{Fall 8}

23-jährige Patientin. Mit 11 Jahren wurde eine Schieloperation durchgeführt, vermutlich bei Strabismus convergens. Diagnose: Dekompensie rende Exophorie, Strabismus divergens et sursumvergens alternans, bevorzugte Fixation rechts. Befunde vor der Therapie: Am Synoptophor beobachtete man eine alternierende Exklusion. Bagolini-Test: Für die Ferne positiv; für die Nähe kam es zeitweise zu einer Exklusion rechts. Eine geringe Amblyopie rechts konnte angenommen werden. Ergebnisse: Eine Stahlnadel im Bereich des Antitragus rechts bewirkte eine deutliche Besserung des Höherstandes, solange die Nadel lag.

\section{Fall 9}

60-jährige Patientin. Diagnose: Amblyopie rechts bei Anisomyopie Strabismus divergens monolateralis rechts. Befunde vor der Therapie: Fernvisus rechts 0,5 mit $-11,5$ Dioptrien, links 1,0 mit $+1,75$ Dioptrien. Therapie: Am ventralen Ohrläppchen rechts wurde eine Laserakupunktur mit der 
Frequenz $9592 \mathrm{~Hz}$ durchgeführt. Ergebnisse: Unmittelbar nach Beginn der Belichtung sah man im alternierenden und einseitigen Abdecktest keine Einstellbewegung. Unmittelbar nach Löschen der Belichtung beobachtete man spontan einen Geradstand.

\section{Fall 10}

13-jährige Patientin. Diagnose: Beidseitige Amblyopie bei Nanophthalmus und Astigmatismus. Strabismus divergens monolateralis rechts. Befunde vor der Therapie: Refraktion: beidseits $+9,0$ comb. cyl. $-3,0$ bei $170^{\circ}$. Fernvisus beidseits 0,2 . Nystagmus bei monokularer Fixation besonders rechts. Therapie: Laserakupunktur am Nasenrücken beidseits mit der Frequenz 20 Hz. Ergebnis: Unmittelbar nach Beginn der Belichtung mit der Laserneedle kam es zu einer deutlichen Verkleinerung des Schielwinkels. Nach wenigen Tagen kam zu einem Rezidiv. Mit je einer Laserneedle am lateralen Lidwinkel rechts (Frequenz 299,75 Hz) und im Bereich des medialen Lidwinkels rechts (Frequenz $10 \mathrm{~Hz}$ ) wurde der Schielwinkel während der Behandlung schnell kleiner.

\section{Fall 11}

61-jährige Patientin. In der Kindheit wurden vier Schieloperationen durchgeführt. Diagnose: Strabismus divergens intermittens rechts. Amblyopie rechts. Befunde vor der Therapie: Fernvisus rechts 0,5 partim; links 1,0 Anisometropie, rechtsbetonte Hypermetropie. In Zykloplegie rechts $+5,5$ comb. cyl. $-1,0$, Achse $6^{\circ}$; links $+2,75$ comb. cyl. $-0,25$, Achse $174^{\circ}$ Therapie: Laserakupunktur am rechten ventralen Ohrläppchen. Ergebnis: Mit der Frequenz 559,5 Hz und $9592 \mathrm{~Hz}$ zeigte sich keine Wirkung auf das Schielen. Mit der Frequenz $20 \mathrm{~Hz}$ wurden die Einstellbewegungen kleiner. Mit einer zusätzlichen Belichtung am linken ventralen Ohrläppchen mit $20 \mathrm{~Hz}$ nahmen die Einstellbewegungen beim Abdecktest noch mehr ab.

\section{Fall 12}

28-jährige Patientin. Diagnose: Status nach Schieloperation. Strabismus monolateralis divergens links und Anisometropie. Befunde vor der Therapie: Linksbetonte Hypermetropie und Astigmatismus. Refraktion in Miose: rechts $+1,25$ comb. $-0,5$, Achse $1^{\circ}$; links $+3,0$ comb. $-1,5$, Achse $4^{\circ}$. Therapie: Beidseits wurde eine Nadel in die Gegend des Antitragus gegeben. Ergebnis: Die Einstellbewegungen blieben aus, solange die Nadeln lagen.

\section{Fall 13}

64-jährige Patientin. Diagnose: Status nach Embolisation eines Aneurysmas der Arteria vertebralis rechts distal vom Abgang des PICA vor 3 Jahren. Vertikale Doppelbilder. Blickrichtungsabhängiger Downbeat-Nystagmus. Befunde vor der Therapie: Die Doppelbilder traten vermehrt bei Blick nach unten und nach beiden Seiten auf. Die Patientin konnte wegen der Doppelbilder ihre Wohnung nicht verlassen. Bei Blick nach links und in Primärposition sah sie stets doppelt. Die Doppelbilder waren weniger ausgeprägt bei Blick nach rechts und nach oben. Bei Vorwärtsneigung des Kopfes (die Patientin schaute dann etwas hinauf) wurde der Nystagmus geringer; die Höhenabweichung des linken Auges war im Schober-Test noch sichtbar.

Therapie: Eine Nadel (Stahl, 0,2 mm) wurde etwas dorsal vom Zentrum des rechten Ohrläppchens gegeben (Abb. 3). Ergebnis: Unmittelbar nach Setzen der Nadel ging der Nystagmus zurück. Die vertikale Abweichung im Schober-Test war unter vergleichbarer Blickrichtung geringer. Die Patientin nahm im Schober-Test und subjektiv eine Besserung der Oszillation wahr. Bei leichter Neigung des Kopfes nach vorn war kein Nystagmus mehr zu sehen. Auch die Patientin nahm wahr, dass sich die Oszillation in dieser Position gebessert hatte. Unter der Aufhebung der Fixation (Untersuchung mit Frenzelbrille) war der Downbeat-Nystagmus weniger ausgeprägt. Die Doppelbilder bei Blick nach links bestanden weiterhin. Nach 15 min wurde die Nadel gezogen. Ein neuer Einstich erfolgte $4 \mathrm{~mm}$ dorsal der ersten Stichstelle. Ergebnis: Der Nystagmus verringerte sich nochmals; im Schober-Test nahm die Patientin weniger Oszillation und eine geringere Höhenabweichung wahr. Die Doppelbilder bei Blick nach links waren noch vorhanden. Nach 15 min wurde die zweite Nadel gezogen. Ergebnis nach einer Woche: Die Patientin gab an, die Sehstörung sei in der Zwischenzeit weniger störend gewesen. In Primärposition sah sie im Schober-Test keine Oszillation. Bei Blick nach unten war ein grobschlägiger Nystagmus von $2 \mathrm{~Hz}$ sichtbar. Therapie: Eine Nadel wurde ins zentrale Ohrläppchen rechts gegeben. Ergebnis: Der Nystagmus wurde unter der Nadel geringer. Nach 25 min wurde die Nadel gezogen.

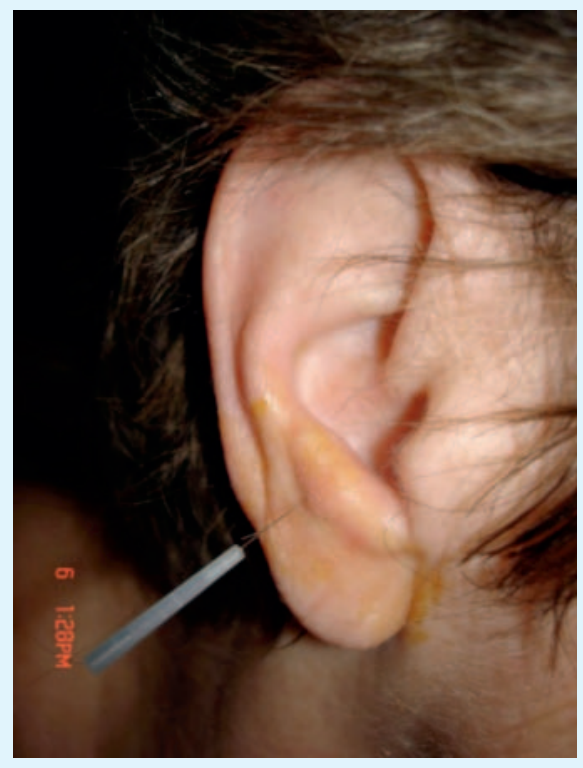

Abb. 3. Fall 13: Eine Nadel wurde dorsal vom Zentrum des Ohrläppchens gegeben.

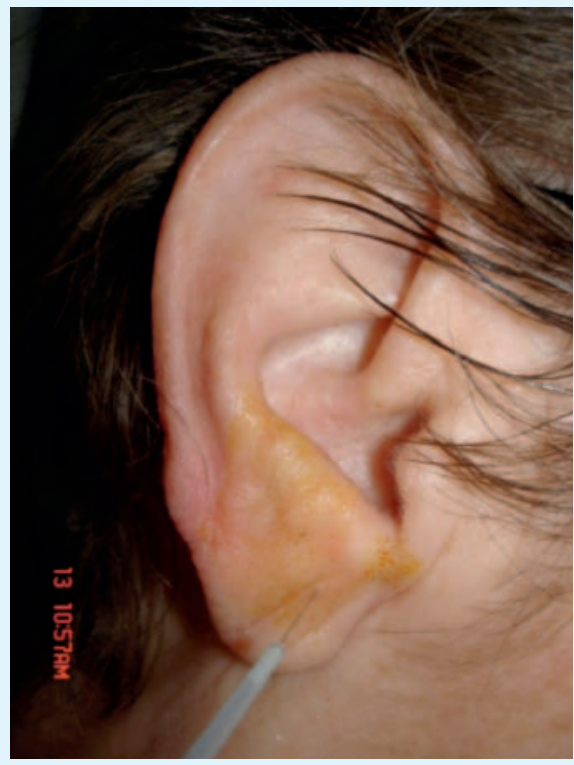

Abb. 4. Fall 13:

Eine neue Nadel an einer neuen Lokalisation wurde kaudal des Antitragus rechts gegeben.

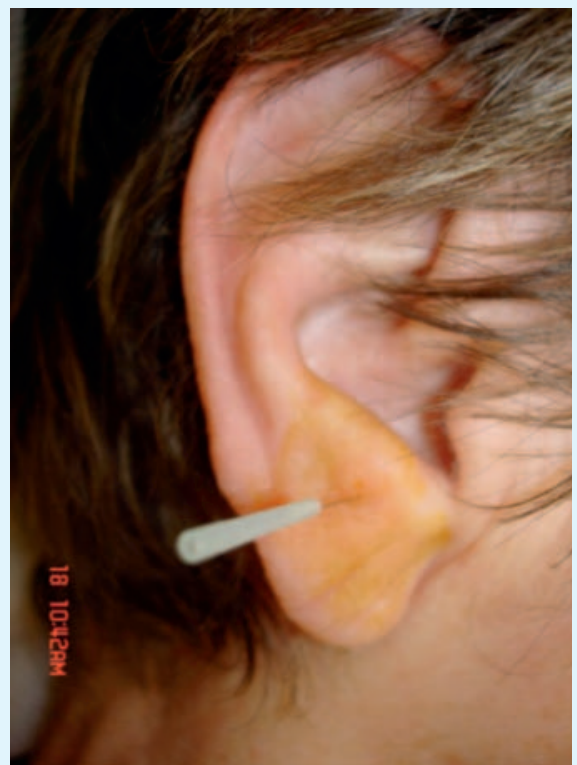

Abb. 5. Fall 13:

Eine neue Nadel an einer neuen Lokalisation (am rechten Ohrläppchen, etwas dorsal vom Zentrum) wurde gegeben. 


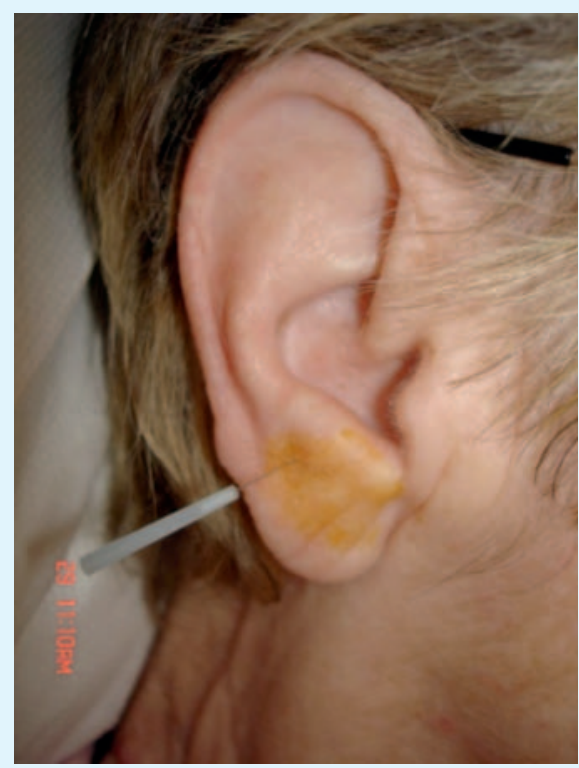

Abb. 6. Fall 13:

Rechts wurde etwas dorsal vom Zentrum der Ohrläppchen eine Nadel gegeben.

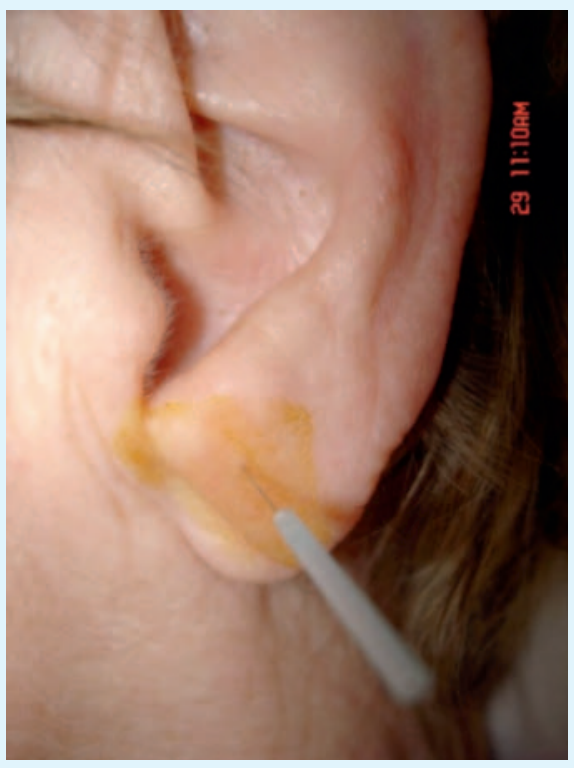

Abb. 7. Fall 13:

Links wurde etwas dorsal vom Zentrum der Ohrläppchen eine Nadel gegeben.
Ergebnis nach 2 Tagen: In der Zwischenzeit bemerkte die Patientin eine Besserung der Sehstörung. Im Schober-Test wurden weniger Oszillationen wahrgenommen als vor der Ohrakupunktur. Bei der Fortbewegung ausserhalb der Wohnung war sie noch unsicher. Befunde: Bei Blick nach unten war ein grobschlägiger Nystagmus zu beobachten. Bei Blick nach links traten Doppelbilder auf. Therapie: Aufgrund des Tinnitus wurde eine Nadel kaudal des Antitragus rechts gegeben (Abb. 4). Die Stelle lag nicht dort, wo bereits gestochen wurde. Ergebnis nach 5 Tagen: Die Sehstörung hatte wieder zugenommen. Therapie: Eine Nadel wurde am rechten Ohr läppchen, etwas dorsal vom Zentrum, an ähnlicher Stelle wie zu Beginn der Akupunkturbehandlung, gegeben (Abb. 5). Ergebnis: Unmittelbar nach dem Einstich wurde der Nystagmus geringer.

2 Tage später: Die Sehstörung war wieder etwas geringer, aber nicht so gut wie nach den vorhergehenden Sitzungen. Therapie: Eine Nadel wurde ins zentrale Ohrläppchen rechts gegeben. Ergebnis nach 5 Tagen: Bei Blick in Primärposition sah die Patientin kaum noch Doppelbilder. Bei Blick nach unten trat ein deutlicher Nystagmus auf. Beim Gehen hatte die Patientin den Eindruck einer Erschütterung. Das binokulare Blickfeld war nun bis $15^{\circ}$ nach rechts und $30^{\circ}$ nach links frei von Doppelbildern. Beidseits wurde etwas kranial vom Zentrum der Ohrläppchen eine Nadel gegeben. Unter der Nadelanwendung konnte sie besser umhergehen. Nach 25 min wurden die Nadeln gezogen. Der Nystagmus war aus der Sicht der Patientin und für den Untersucher geringer als vor der Akupunktur.

Befunde nach 4 Tagen: Die Sehstörungen waren deutlich geringer als vor der Akupunktur. Vor allem war die Blickwendung rechts bis $30^{\circ}$ und links bis $5^{\circ}$ möglich, ohne dass Doppelbilder auftraten. Therapie: Beidseits wurde wiederum etwas dorsal vom Zentrum der Ohrläppchen eine Nadel gegeben (Abb. 6, 7). Befunde: Das Blickfeld war nun rechts bis etwa $20^{\circ}$, links bis $30^{\circ}$ frei von Doppelbildern. Möglicherweise war der Nystagmus besser als vor der Akupunktur.

\section{Fall 14}

33-jährige Patientin. Diagnose: Schielamblyopie. Strabismus divergens monolateralis rechts. Befunde vor der Therapie: Der bestkorrigierte Fernvisus war rechts 0,3 und links 1,0 . Rechts war eine deutlich temporale Fixation zu sehen. Links war die Fixation parazentral. Therapie: Laserakupunktur am rechten ventralen Ohrläppchen mit der Frequenz von $20 \mathrm{~Hz}$ Ergebnis: Während der Belichtung war die Fixation deutlich zentraler (Untersuchung mit dem Ophthalmoskop). Mit der Frequenz $10 \mathrm{~Hz}$ und $40 \mathrm{~Hz}$ am gleichen Ort war keine Wirkung auf die Fixation zu beobachten.

\section{Fall 15}

35-jährige Patientin. Diagnose: Amblyopie links bei hochgradigem Astigmatismus links. Exzentrische Fixation links. Strabismus divergens monolateralis links. Befunde vor der Akupunktur: Links lag ein hochgradiger hypermetroper Astigmatismus (+6 Dioptrien bei $62^{\circ}$ ) vor. Die Fixation links war exzentrisch temporal. Der bestkorrigierte Fernvisus war rechts 0,9 und links 0,2. Abdecktest: Strabismus divergens monolateralis links. Therapie: Laserakupunktur am rechten Ohrläppchen nasal mit der Frequenz $20 \mathrm{~Hz}$. Ergebnis: Die Fixation links war vorübergehend zentral. Unmittelbar nach der Belichtung war die Fixation wieder wie vorher.

\section{Fall 16}

46-jährige Patientin. Diagnose: Amblyopie links ex Anisometropie. Befunde vor der Therapie: Automatisches Refraktometer in Mydriase rechts $+4,0$, comb. $-0,75$, Achse $86^{\circ}$; links $+7,0$, comb $-0,5$, Achse $47^{\circ}$. Fernvisus rechts mit bester Korrektur 1,0 (2 von 4 ) und links 0,3 (2 von 4 ). Fixation rechts zentral. Links nasal 0,5; Papillendurchmesser exzentrisch. Der Foveolarreflex war beidseits sichtbar. Therapie: Eine Nadel wurde ins rech te Ohrläppchen, $5 \mathrm{~mm}$ kaudal des Antitragus und $3 \mathrm{~mm}$ nach okzipital, gegeben. Ergebnis: Nach $10 \mathrm{~s}$ war die Fixation etwa um die Hälfte der vorherigen Distanz in die Nähe des Foveolarreflexes gerückt. Nach 1 min war die Fixation zentral; der Foveolarreflex wurde von der Fixiermarke bedeckt. Der Fernvisus mit $+6,0$ und stenopäischer Lücke war zögernd, möglicherweise langsam zunehmend 0,2 . Nach 2 min wurde die $0,2 \mathrm{~mm}$ dicke Stahlnadel gezogen. Nach $10 \mathrm{~s}$ war die Fixation wieder erkennbar exzentrisch nasal; nach 1 min war die Fixation wieder gleich exzentrisch wie vor der Akupunktur. Der beste Fernvisus mit stenopäischer Lücke war 2 min nach Entfernung der Nadel 0,3 (2 von 4). (Mit Vorsetzen einer stenopäischen Lücke kann man den Einfluss der Refraktion und der brechenden Medien auf die Sehschärfe verringern.)

\section{Fall 17}

28-jährige Patientin. Diagnose: Commotio cerebri und contusio frontotemporal vor 4 Jahren. Dekompensierende Exophorie. Schieloperation vor 1 Jahr. Doppelbilder seit 5 Monaten. Befunde vor der Therapie: Der bestkorrigierte Fernvisus war beidseits voll. Abdecktest: Besonders für die Nähe bestand eine Exophorie; die Einstellbewegung war mit Anstrengung möglich. Akkommodationsbreite beidseits 3-4 Dioptrien. Therapie: Ven tral kranial am Ohrläppchen rechts wurde eine $0,2 \mathrm{~mm}$ dicke Nadel gegeben. Ergebnis: Die Doppelbilder bei der Folgebewegung waren unter der Nadel nach Sekunden nicht mehr feststellbar. Die Einstellbewegung im Abdecktest für die Ferne war verschwunden. Die Besserung hielt nach dem Entfernen der Nadel etwa 17 Tage an. Therapie: Erneut wurde an der gleichen Stelle eine Nadel gegeben, die 15 min liegen blieb. Ergebnis: Die Doppelbilder waren $10 \mathrm{~s}$ nach Legen der Nadel besser.

Die Wirkung der Akupunktur hielt nach Angaben der Patientin knapp 3 Wochen an. Sie konnte besser fusionieren als vor der Akupunktur. Therapie: Bei einer neuen Sitzung wurde eine Nadel kaudal des Antitragus und an der vorher beschriebenen Stelle am rechten Ohr gesetzt. Ergebnis: Die 
Fusion unter der Nadel ist bedeutend leichter möglich als vor dem Einstich der Nadel.

Fall 18

73-jähriger Patient. Diagnose: Abduzensparese rechts seit einigen Tagen. Eine Ursache war nicht feststellbar. Befunde vor der Therapie: Das rechte Auge stand divergent. Der bestkorrigierte Visus war beidseits voll. Therapie: Ohrakupunktur am rechten Ohrläppchen ventral kranial sowie am linken Ohr ventral und dorsal am Ohrläppchen. Ergebnis: Eine Wirkung auf die Abduzensparese liess sich nicht beobachten.

\section{Fall 19}

56-jährige Patientin. Diagnose: Abduzensparese rechts seit 2 Tagen. Insulinpflichtiger Diabetes mellitus seit 8 Jahren. Befunde vor der Therapie: Die Augen standen bei Blick geradeaus (Primärposition) divergent. Der bestkorrigierte Fernvisus war beidseits voll. Therapie: Viermal wurde im Bereich des Antitragus rechts im Zeitraum von 5 Wochen eine Nadel gegeben. Ergebnis: Die Doppelbilder wurden nicht sicher besser. Nach 3 Monaten waren die Doppelbilder verschwunden. Es war unklar, ob die Akupunktur am Antitragus rechts eine Wirkung hatte oder ob es zu einer spontanen Remission kam.

\section{Fall 20}

27-jährige Patientin. Diagnose: Dekompensierende Exophorie sowie Asthenopie. Befunde vor der Therapie: Spontan standen die Augen diver gent. Die Patientin verspürte Kopfschmerzen bei genauem Hinschauen. Therapie: Kranial ventral am rechten Ohrläppchen wurde eine 0,2 mm dicke Nadel gegeben. Ergebnis: Die Einstellbewegung beim Abdecktest war unter der Nadel deutlich besser. 5 Monate später verspürte die Patien tin weniger Kopfschmerzen. Sie sagte, die Akupunktur habe gut gewirkt.

\section{Fall 21}

48-jährige Patientin. Diagnose: Trochlearisparese rechts. Asthenopie beim Schauen in die Nähe. Befunde vor der Therapie: Im Kopfneigetest sah man einen Höherstand rechts über links. Doppelbilder traten besonders in die Nähe auf. Mit 6 PD unten rechts waren die Doppelbilder ausgeglichen. Therapie: Im Abstand von 8 Tagen wurde ventral kranial am rechten Ohr läppchen eine 0,2 mm dicke Nadel gegeben (zweimal) (Abb. 5, mit Dauer nadel). Ergebnis: Im Sehschulstatus konnte eine Besserung der vertikalen Abweichung gesehen werden. Im Abstand einer Woche folgten zwei weitere Akupunkturen an ähnlicher Stelle am rechten Ohr, jeweils mit einer Dauernadel zum Abschluss. Die Patientin benötigte anschliessend nach der Akupunktur keine Prismen mehr.

\section{Fall 22}

73-jähriger Patient. Seit 3 Tagen bemerkte der Patient horizontale Doppelbilder. Diagnose: Abduzensparese rechts. Befunde vor der Therapie: Das rechte Auge stand divergent. Der bestkorrigierte Fernvisus war beidseits voll. Therapie: Eine Nadel wurde am rechten Ohrläppchen ventral kranial gegeben. Ergebnis: Ein Einfluss auf die Abduzensparese konnte nicht festgestellt werden (Abb. 8).

\section{Ergebnisse}

Die Wirkung der Ohrakupunktur mit Nadeln: Bei drei Patienten mit einer Abduzensparese wurde keine Wirkung festgestellt (Fälle 18, 19, 22). Bei den anderen Patienten konnte eine Wirkung festgestellt werden. Die Doppelbilder besserten sich unter dem Einfluss der Akupunktur (Fälle 1-3, 13, 17, 21). Der Schielwinkel besserte sich bei allen Patienten ausser bei den Fällen 18, 19 und 22. Der Nystagmus besserte sich bei den Fällen 1-3 sowie 13. Die dekompensierende Exophorie besserte sich bei den Fällen 4, 5, 8, 11 und 20. Die exzentrische Fixation wurde bei den Fällen 14-16 beeinflusst. (Die Fixationsmarke wurde mit dem Ophthalmoskop während der Akupunktur beobachtet.)

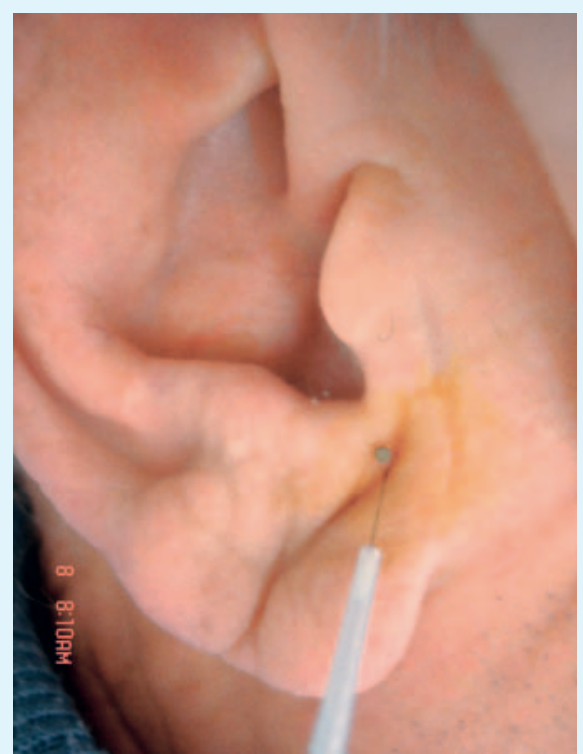

Abb. 8. Fall 22: Vermutlich alte Trochlearisparese.

Bei der Anwendung der Laserneedle zeigte es sich, dass nicht jede Frequenz eine Wirkung entfaltete (Fälle 6, 11, 14). Bei den Patienten mit MS (Fälle 1-3) wurde eine Steigerung der Kraft mitgeteilt; die Patienten konnten besser gehen. Es trat nie eine Zunahme der Doppelbilder, des Schielwinkels oder des Nystagmus unter der Akupunktur oder nach der Akupunktur auf.

Die Wirkung konnte objektiv beobachtet werden; zudem schilderten die Patienten ihre Eindrücke. Es wurde berichtet, dass die Doppelbilder abnahmen oder dass binokulares Sehen ohne Anstrengung möglich sei. Diese Eindrücke vonseiten der Patienten traten oft schon Sekunden nach dem Beginn der Akupunktur auf und waren auch gleichzeitig zu beobachten. Die Dauer der Wirkung war unterschiedlich (nur während der Einwirkung der Akupunktur bis einige Wochen). Bei einem Patienten mit dekompensierender Exophorie trat eine jahrelang dauernde Wirkung auf Laserakupunktur am Ohr mit Geradstand der Augen auf.

\section{Diskussion}

Der Parallelstand wurde bei allen Patienten, die auf die Behandlung mit Akupunktur ansprachen, gestärkt. Es kam nie zu einer Überreaktion. Die Akupunktur führte nie zu einer Zunahme der Doppelbilder oder von anderen Störungen der Augenbewegung, auch nicht $\mathrm{zu}$ einer Zunahme der Beschwerden. Diese Beobachtungen legen eine Stärkung des Fixationssystems und der Koordiantion der Augenmotilität nahe. Gleichzeitig wurde bei mehreren Patienten (1-3) zusätzlich eine Verbesserung des Gehens oder der Müdigkeit beobachtet. Gestärkt wurden also nicht nur das Fixationssystem der Augen, sondern auch andere Funktionen. 
Der Visus wurde bei der Behandlung der Patienten nicht systematisch vor und nach der Behandlung erhoben, sondern meist nur vor der Behandlung.

Zum Mechanismus der Wirkung von Ohrakupunktur und Laserakupunktur am Ohr lässt sich feststellen, dass nicht klar ist, ob eine Verbesserung der Koordination verschiedener Teilfunktionen aufgetreten ist oder ob einzelne gestörte Teilfunktionen selektiv verstärkt und gebessert wurden. Das Fehlen einer Überreaktion und der schnelle Eintritt der Wirkung legen eine funktionelle Wirkung im zentralnervösen System nahe. Eine Wirkung auf die Motorik hätte vielleicht eine Überreaktion hervorgerufen. Die Ergebnisse unter und nach Ohrakupunktur sowie Laserakupunktur hinterliessen indessen den Eindruck des Wiederherstellens eines Normalbefundes, wie aus einem Gedächtnisinhalt.
Der unterschwellige Reiz der Laserneedle konnte auch eine Wirkung ähnlich wie bei der Reizung mit einem Nadelstich hervorrufen. Bei der Nadelakupunktur spielte der Ort des Einstichs eine Rolle. Bei der Anwendung der Laserneedle war bei unserem Apparat die Frequenz entscheidend. Beide Arten der Reizung, Nadel und Laserstrahl, erzielten bei manchen Patienten innerhalb von Sekunden bis Minuten sichtbare Wirkungen auf das Schielen.

\section{Disclosure Statement}

Es bestehen keine Interessenkonflikte.

\section{Literatur}

1 Ercolani M, Poli EG, Zucchini GE: Acupuncture in the treatment of innervation strabismus (Article in Italian). Minerva Med 1982;73:2039-2042.

-2 Kovalsavskii El, Dubrovskaia LA, Gubareva RA, Lazareva LA: Clinical effect of the use of reflexotherapy in children with strabismus and amblyopia (Article in Russian). Vestn Oftalmol 1984;1:28-31.

3 Zhong MQ: Plum-blossom needle therapy in concomitant strabismus. A report of 103 cases. J Tradit Chin Med 1984;4:7-10.

4 Zheng JZ, Wang WM, Chen ZS, Cao RF: Paralytic squint treated with acupuncture. J Tradit Chin Med 1984;4:177-180.

5 Liu C, Wang Y: 81 Cases of Paralytic strabismus treated with acupuncture. J Tradit Chin Med 1993;13:101-102.

6 Yuan X: Clinical observation on treatment of acquired paralytic strabismus by acupuncture plus oral administration of Chinese herbs J Tradit Chin Med 2003;23:188-190.

7 Bricot B: La Reprogrammation Posturale Globale. Montpellier, Édition Sauramps Médical, 1996.

8 Fatrai A, Uhrig S (Hrsg): Chinesische Medizin in der Augenheilkunde. München, Urban und Fischer, 2005.

-9 Zhang SJ, Li SR, Li JS, Liu J, Song RX: Clinical observation on acupuncture for treatment of paralytic strabismus (Article in Chinese). Zhongguo Zhen Jiu 2009;29:799-803.

10 Guo HM, Dong JK, Wang HM, Zhang ZH, Li YX, Wang RX, Liu SQ: Observation on therapeutic effect of acupuncture combined with western medicine on paralytic strabismus (Article in Chinese). Zhongguo Zhen Jiu 2008;28:399-401.

-11 Tian FS, Yang WG, Song HL, Duan HM, Su XY, Li WD: Randomized controlled study on acupuncture for treatment of diabetic paralytic squint (Article in Chinese). Zhongguo Zhen Jiu 2008;28:84-86.

12 Ding SH, Zuo J: Acupuncture for treatment of 40 cases of convergence insufficient asthenopia with exotropia (Article in Chinese). Zhongguo Zhen Jiu 2008;28:345-347.
13 Litscher G, Wang L, Wiesner-Zechmeister M: Specific effects of laser acupuncture on the cerebral circulation. Laser Med Sci 2000;15: 57-62.

14 Litscher G, Wang I, Huber E: Veränderungen cerebraler nahinfrarot-spektroskopischer Parameter während manueller Akupunkturnadelstimulation. Biomed Tech 2002;47:7679.

15 Litscher G, Schwarz G, Sandner-Kiesling A, Hadolt I, Eger E: Effects of acupuncture on the oxygenation of cerebral tissue. Neurol Res 1998;20(suppl 1):28-32.

16 Kawakita K, Shinbara H, Imai K, Fukuda F, Yano T, Kurijama K: How do acupuncture and moxibustion act? - Focusing on the progress in Japanese acupuncture research. J Pharmacol Sci 2006;100:443-459.

17 Reshetniak VK, Dolgikh VG, Durinian RA: Modulating influence of the $2 \mathrm{~d}$ somatosensory cortex of the effects of electroacupuncture on the trigeminal nuclei (Article in Russian). Biull Eksp Biol Med 1985;100:264-265.

18 Bai L, Yan H, Li L, Qin W, Chen P, Liu P, Gong Q, Liu Y, Tian J: Neural specificity of acupuncture stimulation at pericardium 6: evidence from an FMRI study. J Magn Reson Imaging 2010;31:71-77.

19 Ho TJ, Duann JR, Chen CM, Chen JM, Shen WC, Lu TW, Liao JR, Lin ZP, Shaw KN, Lin JG: Temporally shifted hemodynamic response model helps to extract acupuncture-induced functional magnetic resonance imaging blood oxygenation-level dependent activities. Chin Med J (Engl) 2009;122:823829.

21 Zhang Y, Qin W, Liu P, Tian J, Liang J, Von Deneen KM, Liu Y: An fMRI study of acupuncture using independent component analysis. Neurosci Lett 2009;449:6-9.

22 Qin W, Tian J, Bai L, Pan X, Yang L, Chen P, Dai J, Al L, Zhao B, Gong Q, Wang W, Von Deenen KM, Liu Y: FMRI connectivity analysis of acupuncture effects on an amygdala-associated brain network. Mol Pain 2008;4:55.
$23 \mathrm{Wu} \mathrm{Y}$, Jin $\mathrm{Z}$, Li K, Lu ZL, Wong V, Han TL, Zheng H, Caspi O, Liu G, Zeng YW, Zou LP: Effect of acupuncture on the brain of children with spastic cerebral palsy using functional neuroimaging (fMRI). J Child Neurol 2008;23:1267-1274. Comment in J Child Neurol 2009;24:1324-1325; author reply $1325-1326$.

24 Xiao YY, Du L, Hong BK, et al: Study on fMRI brain map in patients undergoing needling of Zusanli (ST36) by reinforced method (Article in Chinese). Zhongguo Zhong Xi Yi Jie He Za Zhi 2008;28:122-125.

25 MacPherson $\mathrm{H}$, Green G, Nevado A, Lythgoe MF, Lewith G, Devlin R, Haselfoot $\mathrm{R}$, Asghar AU: Brain imaging of acupuncture comparing superficial with deep needling. Neurosci Lett 2008;434:144-149.

26 Ho TJ, Duann JR, Chen CM, Chen JH, Shen WC, Lu TW, Liao JR, Lin JG: Carryover effects alter fMRI statistical analysis in an acupuncture study. Am J Chin Med 2008, 36:55-70.

27 Xu FM, Xie P, Lu FJ, Mou J, Li YM, Zhao JN, Chen WJ, Gong QY, Zhao LB, Liu QJ, Shen L, Zhai H, Yang DY: Study of corresponding areas of the liver and lung channels in brain with $\mathrm{fMRI}$ (Article in Chinese). Zhongguo Zhen Jiu 2007;27:749-752.

28 Strudwick MW, Wilson SJ, Stephen J: Automatic syringe driver for acupoint injection in functional MRI studies: a case for quantified point injection in neuroimaging studies of acupuncture. Expert Rev Med Devices 2007;4:815-819.

29 Napadow V, Fettner N, Liu J, Li M, Kwong KK, Vangel M, Makris N, Audette J, Hui KKS: Hypothalamus and amygdala response to acupuncture stimuli in Carpal Tunnel Syndrome. Pain 2007;130:254-266.

30 Moncayo R, Rudisch A, Diemling $M$, Kremser C: In vivo visualisation of the anatomical structures related to the acupuncture points Dai mai and Shen mai by MRI: a single-case pilot study. BMC Med Imaging 2007;7:4. 
-31 Chae Y, Park HJ, Hahm DH, Hong M, Ha E, Park HK, Lee H: fMRI review on brain responses to acupuncture: the limitations and possibilities in traditional Korean acupuncture. Neurol Res 2007;29(suppl 1):S42-48.

32 Litscher G: Bioengineering assessment of acupuncture, part 4: functional magnetic resonance imaging. Crit Rev Biomed Eng 2006;34:327-345.

33 Fang SH, Zhang SZ, Liu H: Study on brain response to acupuncture by functional magnetic resonance imaging-observation on 14 healthy subjects (Article in Chinese). Zhongguo Zhong Xi Yi Jie He Za Zhi 2006;26:965-968.

34 Li G, Jack CR Jr, Yang ES: An fMRI study of somatosensory-implicated acupuncture points in stable somatosensory stroke patients. J Magn Reson Imaging 2006;24:10181024.

35 Li K, Shan B, Xu J, Liu H, Wang W, Zhi L, Li K, Yan B, Tang X: Changes in $\mathrm{AMRI}$ in the human brain related to different durations of manual acupuncture needling. J Altern Complement Med 2006;12:615-623.

36 White P, Lewith G: Could neuroimaging help us to interpret the clinical effects of acupuncture? Bundesgesundheitsblatt Gesundheitsforschung Gesundheitsschutz 2006;49:743748 .
Li G, Ng MC, Wong KK, Luk KD, Yang ES Spinal effects of acupuncture stimulation assessed by proton density-weighted functional magnetic resonance imaging at 0,2 $\mathrm{T}$. Magn Reson Imaging 2005;23:995-999.

8 Jeun SS, Kim JS, Kim BS, Park SD, Lim EC, Choi GS, Choe BY: Acupuncture stimulation for motor cortex activities: a 3T fMRI study. Am J Chin Med 2005;33:573-578.

39 Yan B, Li K, Xu J, Wang W, Li K, Liu H, Shan B, Tsang X: Acupoint-specific fMRI patterns in human brain. Neurosci Lett 2005; 383:236-240.

40 Lee JD, Chon JS, Jeong HK, Kim HJ, Yun M, Kim DY, Kim DI, Parl CI, Yoo HS: The cerebrovascular response to traditional acupuncture after stroke. Neuroradiology 2003;45:780-784.

41 Kong J, Ma L, Gollub RL, Wei L, Yang X, Li D, Weng X, Jia F, Wang C, Li F, Li R, Zhuang D: A pilot study of functional magnetic resonance imaging of the brain during manual and electroacupuncture stimulation of acupuncture point ( $\mathrm{Li} 4 \mathrm{Hegu}$ ) in normal subjects reveals differential brain activation between methods. J Altern Complement Med 2002;8:411-419.
42 Siedentopf CM, Golaszewski SM, Mottaghy FM, Ruff CC, Felber S, Schlager A: Functional magnetic resonance imaging detects activation of the visual association cortex during laser acupuncture of the foot in humans. Neurosci Lett 2002;327:53-56.

43 Wu MT, Hsieh JC, Xiong J, Yang CF, Pan HB, Chen YC, Tsai G, Rosen BR, Kwong KK Central nervous pathway for acupuncture stimulation: localization of processing with functional MR imaging of the brain-preliminary experience. Radiology 1999;212:133141.

44 Cao XD, Xu SF, Lu WX: Inhibition of sympathetic nervous system by acupuncture. Acupunct Ther Res 1983;8:25-35.

45 Imich D, Beyer A: Neurobiologische Grundlagen der Akupunkturanalgesie. Schmerz 2002; 16:93-102.

46 Litscher G: Computer-based quantification of traditional Chinese-, ear- and Korean hand acupuncture: needle-induced changes of regional cerebral blood flow velocity. Neurol Res 2002;24:377-380. 